\title{
Continuous periprosthetic bone loss but preserved stability for a collum femoris-preserving stem: follow-up of a prospective cohort study of 21 patients with dual- energy X-ray absorptiometry and radiostereometric analysis with minimum 8 years of follow-up
}

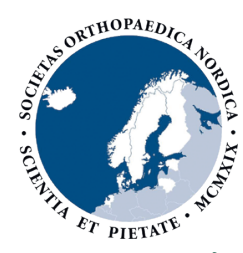

Acta Orthopaedica

\author{
Andreas NYSTRÖM, Demostenis KIRITOPOULOS, Hans MALLMIN, \\ and Stergios LAZARINIS
}

Department of Surgical Sciences/Section of Orthopaedics, Uppsala University, Uppsala, Sweden

Correspondence: andreas.nystrom@surgsci.uu.se

Submitted 2021-04-29. Accepted 2021-11-18.

Background and purpose - We previously described a decrease in bone mineral density (BMD) in the calcar region 2 years after insertion of the collum femoris-preserving (CFP) stem, but the implants were stable. Now we have examined the long-term changes in periprosthetic BMD and stability of the CFP stem.

Patients and methods - We conducted a minimum 8-year follow-up of 21 patients from our original investigation. We examined periprosthetic BMD by dual-energy X-ray absorptiometry (DEXA) and implant stability by radiostereometric analysis (RSA).

Results - Between 2 and 8 years 1 stem was revised due to aseptic loosening. Between 2 and 8 years we found a $14 \%$ (95\% confidence interval $[\mathrm{CI}] 9-19)$ reduction in BMD in Gruen zone 6 and 17\% (CI 6-28) in Gruen zone 7. From baseline the reduction in BMD was 30\% (CI 23-36) in Gruen zone 6,39\% (CI 31-47) in Gruen zone 7, and 19\% (CI 14-23) in Gruen zone 2. Between 2 and 8 years, RSA (n $=17$ ) showed a mean translation along the stem axis of 0.02 $\mathrm{mm}(\mathrm{CI}-0.02$ to 0.06$)$ and a mean rotation around the stem axis of $0.08^{\circ}$ (CI -0.26 to 0.41 ). From baseline mean subsidence was $0.07 \mathrm{~mm}(\mathrm{CI}-0.16$ to 0.03$)$ and mean rotation around the stem axis was $0.23^{\circ}$ ( $\mathrm{CI}-0.23$ to 0.68$)$ at 8 years.

Interpretation - There was continuous loss of proximomedial BMD at 8 years while the CFP stem remained stable. Proximal periprosthetic bone loss cannot be prevented by this stem.
A cause for concern after total hip arthroplasty (THA) is the loss of periprosthetic bone mineral density (BMD) during the first year after surgery (1-3). If continued, this loss could increase the risk of aseptic implant loosening, periprosthetic fractures, or both (4). Few long-term studies have investigated periprosthetic BMD. Moreover, the results of these studies are inconsistent. While some authors report continuous proximal bone loss $(5,6)$ others have found restoration of BMD (7). To prevent BMD loss and sustain good proximal bone stock for future revision, short stems have been introduced (8). In the original study on the CFP stem at our institution (3) we reported a $31 \%$ reduction of BMD in Gruen zone 7 at 1 year post-surgery, despite the proposed proximal load transfer of this stem. We conducted a long-term follow-up of our CFP study (also referred to as the "original study") for periprosthetic BMD, implant stability, radiographic evaluation, and clinical results.

\section{Patients and methods \\ Study design and population \\ Original study}

This study was a prospective cohort study of the uncemented, short CFP stem in patients with primary osteoarthritis of the hip (OAH). Details on inclusion and exclusion criteria, the screening process, and patient characteristics have already been reported (3).

Patients with primary $\mathrm{OAH}$ and indication for uncemented THA were eligible for inclusion. Consecutive recruitment took place from March 2008 to March 2009. 


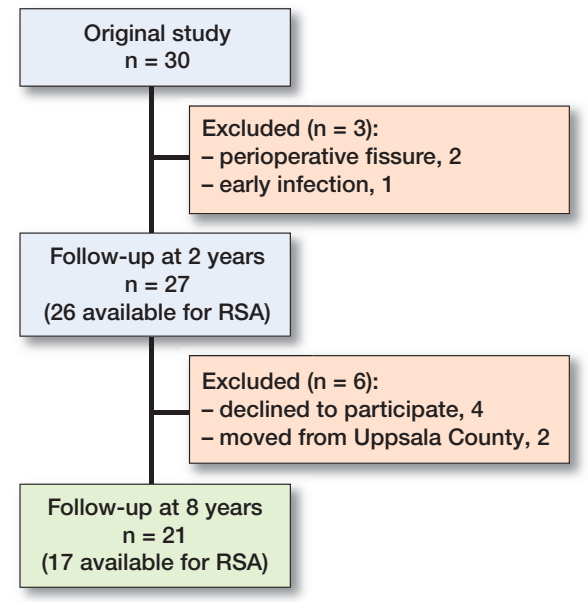

Figure 1. CONSORT flowchart. Numbers represent patients available for follow-up.

30 patients (18 women) with a mean age of 56 years (42-65) were recruited. BMI was 27 (20-35). They were scheduled for THA with the uncemented CFP stem and the uncemented trabeculae-oriented pattern (TOP) cup (Waldemar Link GmbH \& Co. KG, Hamburg, Germany). Patients were followed up for 2 years with dual-energy X-ray absorptiometry (DEXA), radiostereometric analysis (RSA), the Harris Hip Score (HHS), and plain radiography.

3 patients were excluded during the original study: 1 due to revision because of early, deep infection and 2 because they received a different type of femoral stem for perioperative fissures of cortical bone. RSA was not possible for 1 patient for technical reasons, leaving 27 patients ( 26 for RSA).

\section{Present study}

We performed a long-term follow-up of the cohort of the original CFP study by Lazarinis et al. (3).

Exclusion criteria for the follow-up investigation were revision surgery performed on the ipsilateral hip, deceased, having moved from Uppsala County, lost to follow-up, or otherwise unable to participate.

Of the 27 patients from the original study, 6 were not available for this study (Figure 1). Of these 6 patients, 3 had undergone cup revision surgery but were perioperatively found to have well-fixed stems. 1 patient was revised due to stem and cup loosening. All revisions were due to aseptic loosening and the remaining 2 patients reported no implant-related problems but declined further participation.

This study included 21 patients (10 women) with a median age of 64 years (55-73) and a median BMI of 27 (20-32).

\section{Implant and surgery}

2 experienced surgeons performed the surgery. Details on the surgical procedure and implants are available in the original article (3).

\section{Dual-energy X-ray absorptiometry (DEXA)}

DEXA scans of the operated hip were performed by 1 of 2 authors (AN, DK) using a Prodigy Advance system (GELunar, Madison, WI, USA). We analyzed periprosthetic BMD in the 7 zones of Gruen with the dedicated software provided by the manufacturer. The precision at our institution for periprosthetic BMD measurements has previously been reported (coefficient of variation ranges from $1.1 \%$ to $5.7 \%$ ) (3).

\section{Radiostereometric analysis (RSA)}

The tantalum marking of implants and bone perioperatively is described in the original article (3). Mean errors of rigid body fitting were $<0.35$ for all but 1 of the included rigid bodies (0.39) and condition numbers were $<90$ for all but 1 (127).

\section{Clinical and radiographic outcome}

The HHS was used to evaluate hip function. Standard pelvic, anteroposterior, and lateral hip digital radiographs were obtained and assessed for radiolucency and subsidence by AN .

\section{Statistics}

The size of the original cohort was determined by a power analysis of the primary endpoints of the original study: periprosthetic BMD in Gruen zone 7 measured with DEXA and subsidence measured with RSA (3). According to this analysis 20 patients would be sufficient to detect a BMD difference of 1 standard deviation in Gruen zone 7 and a subsidence of more than $2 \mathrm{~mm}$ with a power of $80 \%$, if a 2-tailed alpha of 0.05 were applied. Statistical calculations were performed with SPSS Statistics software version 27 (IBM Corp, Armonk, NY, USA).

Standard descriptive statistics were used to summarize the data as appropriate.

The within-subjects effect was calculated using a repeated measures ANOVA. The Greenhouse-Geisser adjustment was applied if sphericity was violated according to Mauchly's test. In hypothesis testing the level of significance was set to 5\% (p $<0.05$ ), and $95 \%$ confidence intervals (CIs) were calculated.

\section{Ethics, funding, and potential conflicts of interests}

The study was performed in compliance with the provisions of the Helsinki Declaration. All patients gave written informed consent, and approval was given by the Regional Ethics Committee in Uppsala (Dnr 2007/105/2). AN received a grant from the foundation Tore Dahléns minne and Stenholms foundation at Norrlands nation. None of the authors have any conflicts of interest.

\section{Results}

\section{Dual-energy $X$-ray absorptiometry}

Between 2 and 8 years we found a 14\% (CI 9-19) reduction in BMD in Gruen zone 6 and 17\% (CI 6-28) in Gruen zone 


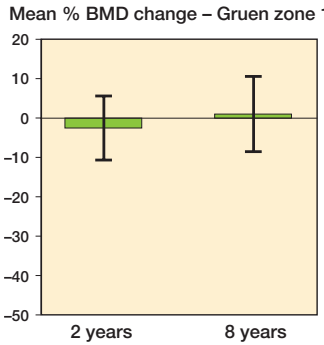

Mean \% BMD change - Gruen zone 2

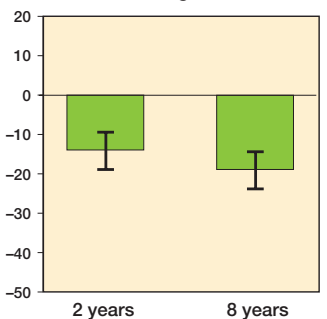

Mean \% BMD change - Gruen zone 3

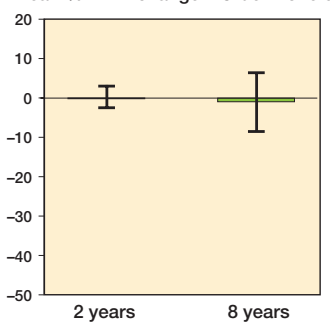

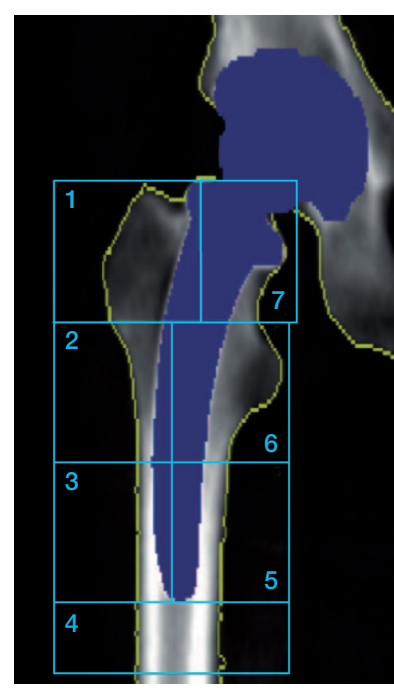

Mean \% BMD change - Gruen zone 4

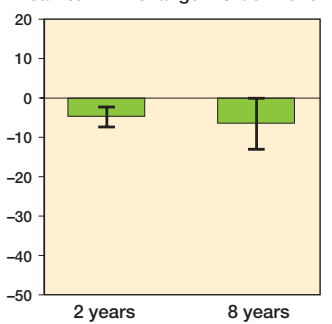

Mean \% BMD change - Gruen zone 7

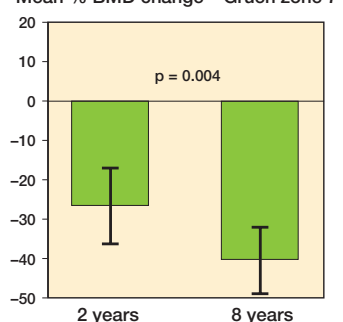

Mean \% BMD change - Gruen zone 6

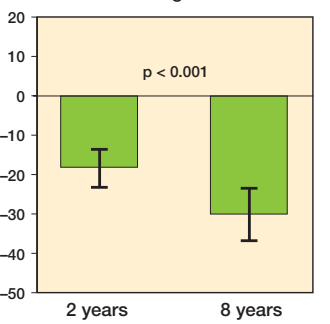

Mean \% BMD change - Gruen zone 5

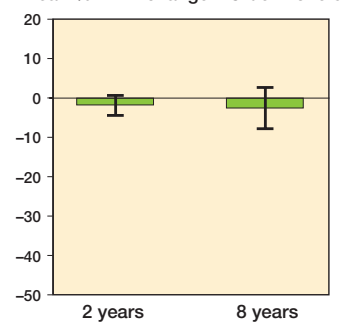

Figure 2. Changes in periprosthetic bone mineral density from baseline $(n=21)$. P-values represent significance in change between 2 and 8 years (above bars). Error bars represent $95 \%$ confidence intervals.

Table 1. Mean BMD $\left(\mathrm{g} / \mathrm{cm}^{2}\right)$ and $(95 \% \mathrm{Cl})$ at the different time points for Gruen zones 1-7

\begin{tabular}{lcccc}
\hline $\begin{array}{l}\text { Gruen } \\
\text { zone }\end{array}$ & 2 days & 1 year & 2 years & 8 years \\
\hline 1 & $0.81(0.73-0.88)$ & $0.77(0.68-0.87)$ & $0.79(0.69-0.89)$ & $0.82(0.71-0.93)$ \\
2 & $1.46(1.37-1.54)$ & $1.29(1.17-1.41)$ & $1.26(1.14-1.38)$ & $1.19(1.07-1.30)$ \\
3 & $2.18(2.07-2.29)$ & $2.17(2.08-2.27)$ & $2.18(2.08-2.28)$ & $2.15(1.97-2.33)$ \\
4 & $2.08(1.95-2.20)$ & $1.99(1.86-2.12)$ & $1.98(1.84-2.13)$ & $1.94(1.75-2.13)$ \\
5 & $2.20(2.10-2.30)$ & $2.14(2.05-2.23)$ & $2.15(2.06-2.25)$ & $2.14(2.00-2.27)$ \\
6 & $1.70(1.61-1.78)$ & $1.38(1.27-1.50)$ & $1.39(1.26-1.51)$ & $1.19(1.04-1.35)$ \\
7 & $1.55(1.46-1.65)$ & $1.08(0.94-1.22)$ & $1.14(0.98-130)$ & $0.94(0.77-1.11)$ \\
\hline
\end{tabular}

$\mathrm{BMD}=$ bone mineral density

Table 2. Migration from baseline at 8 years as measured with radiostereometric analysis in 17 available patients ${ }^{\text {a }}$

\begin{tabular}{lccc}
\hline Factor & Mean & Range & $95 \% \mathrm{Cl}$ \\
\hline X-translation $(\mathrm{mm})$ medial+/lateral- & -0.031 & -0.37 to 0.44 & -0.12 to 0.056 \\
Y-translation $\mathbf{b}(\mathrm{mm})$ proximal+/distal- & -0.066 & -0.43 to 0.34 & -0.16 to 0.029 \\
Z-translation $(\mathrm{mm})$ anterior+/posterior- & 0.044 & -0.58 to 0.46 & -0.073 to 0.16 \\
X-rotation $\left({ }^{\circ}\right)$ anterior tilt+/posterior tilt- & -0.012 & -1.20 to 0.59 & -0.23 to 0.20 \\
Y-rotation $\left({ }^{\circ}\right)$ retroversion+/anteversion- & 0.23 & -1.32 to 1.91 & -0.23 to 0.68 \\
Z-rotation $\left({ }^{\circ}\right)$ valgus+/varus- & 0.036 & $-0.39-0.64$ & $-0 . ? ?$ to 0.17 \\
\hline
\end{tabular}

${ }^{a}$ RSA could not be performed in 4 of the patients for technical reasons.

b Distal translation along the $\mathrm{Y}$-axis is equivalent to subsidence of the stem.

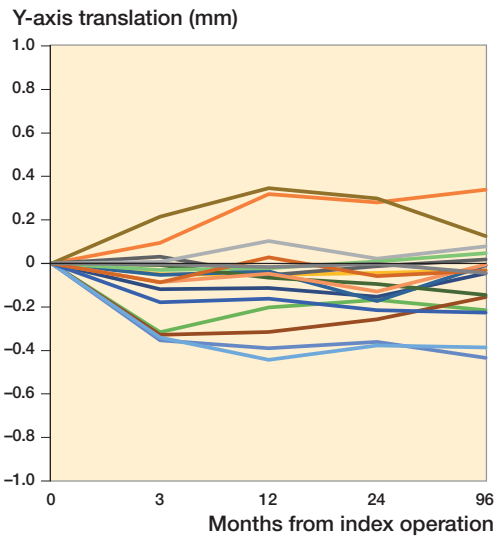

Figure 3. Subsidence pattern of all stems up to 8 years measured as translation along the $\mathrm{Y}$-axis in $\mathrm{mm}$ at the different time points $(\mathrm{n}=17)$.

7 while changes in BMD in Gruen zones 1-5 were minor. From baseline there was a $19 \%$ (CI 14-23) reduction in BMD in Gruen zone $2,30 \%$ (CI 23-36) in Gruen zone 6 and 39\% (CI 31-47) in Gruen zone 7 at the 8-year follow-up (Figure 2, Table 1).

\section{Radiostereometric analysis}

Between 2 and 8 years, 3 stems displayed rotation around the $\mathrm{Y}$-axis exceeding the precision error of the original study $\left(0.83^{\circ}\right)$, with a maximum of $1.7^{\circ}$ in retroversion, and 5 stems displayed translation along the Y-axis exceeding the precision error of the original study $(0.14 \mathrm{~mm})$ with a maximum of 0.17 $\mathrm{mm}$ of subsidence. Mean translation along the Y-axis was $0.02 \mathrm{~mm}(\mathrm{CI}-0.02$ to 0.06$)$ and mean rotation around the stem axis was $0.08^{\circ}$ (CI -0.26 to 0.41 ) from 2 to 8 years.

At 8 years, translation along and rotation around the $\mathrm{X}-$, Y-, and Z-axis was small compared with baseline. 6 stems displayed rotation around the $\mathrm{Y}$-axis exceeding the precision error of the original study with a maximum of $1.9^{\circ}$ in retroversion. 8 stems displayed translation along the $\mathrm{Y}$-axis exceeding the precision error of the original study with a maximum of $0.43 \mathrm{~mm}$ of subsidence. From baseline, mean translation along the Y-axis was $-0.07 \mathrm{~mm}$ (CI -0.16 to 0.03 ) and mean rotation around the stem axis was $0.23^{\circ}(\mathrm{CI}-0.23$ to 0.68$)$ at 8 years (Table 2, Figure 3 ).

\section{Clinical and radiographic outcome}

The mean HHS at 8 years was 95 (82-100) compared with 52 (24-77) preoperatively and $99(92-100)$ at 2 years. Radiologically, there 
was no sign of stem subsidence or rotation, but 1 patient displayed a radiolucent line along the proximolateral margin. 2 stems had been revised, 1 during the original study due to early, deep infection and 1 between 2 and 8 years for aseptic loosening.

\section{Discussion}

We confirmed 2 main findings concerning periprosthetic bone loss. One finding showed that periprosthetic BMD around the CFP stem did not recover between 2 and 8 years. On the contrary, there was a continuous loss of BMD, which was statistically significant in the proximomedial parts of the femur. The second finding is that the CFP stem remained stable with only minor micromotion as measured by RSA.

The highest rate of periprosthetic BMD loss after uncemented THA has been observed in the first year after surgery $(9,5,10)$, as we also reported in our original study (3). Our findings in the present study are in line with those of several prospective studies on uncemented, conventional stems showing plateauing or even continuous loss of proximal periprosthetic

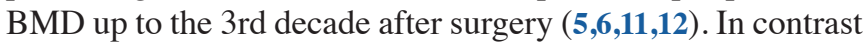
to this, in an RCT on 80 female patients receiving 1 of 4 uncemented conventional stems, a recovery with increasing BMD was seen between 2 and 10 years postoperatively (7). According to Wolff's law, stress shielding accounts for reduction in proximal BMD as load is shifted from the proximal part of the femur, through the rigid implant to cortical bone in the diaphysis $(\mathbf{1 3}, \mathbf{1 4})$. Although the clinical implication of proximal periprosthetic BMD loss remains unclear, short stems have been introduced into surgical practice with the aim of reducing BMD loss $(\mathbf{1 5}, 16)$. Ultra-short stems have shown BMDsparing effects in the short to medium term $(\mathbf{1 7}, \mathbf{1 8})$. However, the pattern of change in periprosthetic BMD depends on stem design and some stems (e.g., the CFP stem) show the same pattern of BMD loss as conventional stems $(\mathbf{1 6 , 1 9 , 2 0 )}$. The position and size of the Gruen zones are defined in relation to the length of the implanted stem. Thus, the Gruen zones differ considerably between an ultra-short and a conventional stem, not only in size but also in position in the proximal femur. There are only a few long-term studies of short stems. A prospective study of an ultra-short stem (Proxima, DePuy, Leeds, UK) showed no proximal BMD loss (Gruen zones 1 and 7) after 14 to 17 years compared with preoperative BMD (21). Long-term studies of the CFP stem are scarce. The few available studies show good clinical results but lack data on BMD measured with DEXA $(22,23)$. A prospective study with long-term BMD results of the CFP stem has been performed in which quantitative computed tomography was used up to 7 years postoperatively (20). In keeping with our study, the authors found no restoration of proximal periprosthetic BMD.

A recently published, prospective, short-term study confirmed the results from our original report of the CFP stem showing good implant stability (24). Our study is the first to report on long-term RSA data on the CFP stem. The early findings of good stability remain, which, combined with excellent clinical outcome, support the observation that small early micromotion on RSA indicates a low late revision rate (25). It also shows that periprosthetic BMD loss does not affect implant stability in the long run. This could indicate that the implant, because of its tri-planar stability, is acting more or less as a conventional stem.

1 patient in the original study showed subsidence of the stem of $1.9 \mathrm{~mm}$ at 2 years. Unfortunately, no RSA data could be obtained for this patient at 8 years for technical reasons; however, plain radiographs showed no further subsidence from 2 to 8 years. BMD loss for this patient was below average for the cohort. The patient had no symptoms, a score of 100 on the HHS and the stem was clinically stable at 8 years.

The original study was designed with the power to evaluate effects of BMD and implant stability, not clinical and radiologic outcome, and implant survival. The long-term clinical outcome for HHS in our cohort is comparable to what has been described for short and conventional uncemented stems in THA (26-28).

2 stems from the original cohort were revised, rendering an 8 -year stem survival of $93 \%$ in the present prospective cohort study in a per-protocol analysis.

Other medium- to long-term retrospective studies of the CFP stem report similar rates of 5-10-year survival of approximately $95 \%$, which is slightly lower than conventional uncemented stems (29-31). Regrettably, these studies lack information on BMD and implant stability from RSA.

The strengths of our study are the prospective design and the combination of DEXA and RSA, making it possible to evaluate whether the observed stress shielding has an impact on implant stability. To our knowledge there are no other prospective studies on BMD and implant stability on the CFP stem.

Our study has weaknesses. First, 6 patients were lost to follow-up for various reasons. Nevertheless, we could obtain information on the revision status of these patients. Notably, only 1 patient had undergone stem revision (in addition to cup revision) due to aseptic loosening. In 3 of these patients, cup revision had been performed and the stems were found to be well fixed perioperatively. For technical reasons, RSA data could not be obtained in 4 patients. However, in RSA studies issues with unstable markers, insufficient quality of images, and difficulties in pairing of markers are well known and loss of investigations is to be expected (32).

Second, the reduced cohort size at 8 years might introduce a type II error making us unable to detect a continuous loss of periprosthetic BMD in other Gruen zones as well as in zones 6 and 7 . However, the initial power calculation indicated that 20 patients would be sufficient to detect a BMD loss of 1 standard deviation at Gruen zone 7 (3). BMD changes below that level were not considered clinically relevant, although the minimal clinically relevant BMD difference is unknown. 
Because different stem design within the group of short or ultra-short stems has resulted in a different pattern of bone remodeling and because the long-term clinical implications of periprosthetic BMD loss remain unclear, further studies are necessary to evaluate these issues (33).

Our findings suggest that the CFP stem shows good clinical results and stable fixation, but continuous proximal BMD loss up to 8 years postoperatively. The design with suggested proximal load transfer does not seem to result in lower stress shielding than conventional uncemented stems.

SL and HM planned the study. AN and DK collected the data and performed DEXA analyses. SL performed RSA analyses. AN and SL performed statistical analyses. AN wrote the first draft. All authors revised the manuscript.

The authors would like to thank Monika Gelotte for excellent assistance with RSA and radiology.

Acta thanks Janus Duus Christiansen and Marc J Nieuwenhuijse for help with peer review of this study.

1. Ang K C, Das De S, Goh J C, Low S L, Bose K. Periprosthetic bone remodelling after cementless total hip replacement: a prospective comparison of two different implant designs. J Bone Joint Surg Br 1997; 79(4): 675-9. doi: 10.1302/0301-620x.79b4.7410.

2. Wolf O, Mattsson P, Milbrink J, Larsson S, Mallmin H. Periprosthetic bone mineral density and fixation of the uncemented CLS stem related to different weight bearing regimes: a randomized study using DXA and RSA in 38 patients followed for 5 years. Acta Orthop 2010; 81(3): 28691. doi: 10.3109/17453674.2010.487238.

3. Lazarinis S, Mattsson P, Milbrink J, Mallmin H, Hailer N P. A prospective cohort study on the short collum femoris-preserving (CFP) stem using RSA and DXA: primary stability but no prevention of proximal bone loss in 27 patients followed for 2 years. Acta Orthop 2013; 84(1): 32-9. doi: 10.3109/17453674.2013.765623.

4. Lindahl H. Epidemiology of periprosthetic femur fracture around a total hip arthroplasty. Injury 2007; 38(6): 651-4. doi: 10.1016/j. injury.2007.02.048.

5. Bodén H S G, Sköldenberg O G, Salemyr M O F, Lundberg H-J, Adolphson P Y. Continuous bone loss around a tapered uncemented femoral stem: a long-term evaluation with DEXA. Acta Orthop 2006; 77(6): 877-85. doi: 10.1080/17453670610013169.

6. Peitgen D S, Innmann M M, Merle C, Gotterbarm T, Moradi B, Streit M R. Periprosthetic bone mineral density around uncemented titanium stems in the second and third decade after total hip arthroplasty: a DXA study after 12, 17 and 21 years. Calcif Tissue Int 2018; 103(4): 372-9. doi: 10.1007/s00223-018-0438-9.

7. Karachalios T, Tsatsaronis C, Efraimis G, Papadelis P, Lyritis G, Diakoumopoulos G. The long-term clinical relevance of calcar atrophy caused by stress shielding in total hip arthroplasty. J Arthroplasty 2004; 19(4): 469-75. doi: 10.1016/j.arth.2003.12.081.

8. Khanuja H S, Banerjee S, Jain D, Pivec R, Mont M A. Short boneconserving stems in cementless hip arthroplasty. J Bone Joint Surg Am 2014; 96(20): 1742-52. doi: 10.2106/JBJS.M.00780.

9. Venesmaa P K, Kröger H P J, Miettinen H J A, Jurvelin J S, Suomalainen O T, Alhava E M. Monitoring of periprosthetic BMD after uncemented total hip arthroplasty with dual-energy X-ray absorptiometry: a 3-year follow-up study. J Bone Miner Res 2001; 16(6): 1056-61. doi: 10.1359/jbmr.2001.16.6.1056
10. Christiansen J D, Laursen M B, Ejaz A, Nielsen P T. Bone remodelling of the proximal femur after total hip arthroplasty with 2 different hip implant designs: 15 years follow-up of the thrust plate prosthesis and the Bi-Metric stem. Hip Int 2018; 28(6): 606-12. doi: $10.1177 / 1120700018755371$.

11. Panisello J J, Herrero L, Canales V, Herrera A, Martínez A A, Mateo J. Long-term remodeling in proximal femur around a hydroxyapatitecoated anatomic stem: ten years densitometric follow-up. J Arthroplasty 2009; 24(1): 56-64. doi: 10.1016/j.arth.2007.12.017.

12. Tapaninen T, Kröger H, Venesmaa P. Periprosthetic BMD after cemented and uncemented total hip arthroplasty: a 10-year follow-up study. J Orthop Sci 2015; 20(4): 657-62. doi: 10.1007/s00776-015-07228

13. Oh I, Harris W H. Proximal strain distribution in the loaded femur: an in vitro comparison of the distributions in the intact femur and after insertion of different hip-replacement femoral components. J Bone Joint Surg Am 1978; 60(1): 75-85.

14. Frost H M. A 2003 update of bone physiology and Wolff's Law for clinicians. Angle Orthod 2004; 74(1): 3-15. doi: 10.1043/00033219(2004)074<0003:AUOBPA>2.0.CO; 2.

15. Pipino F, Molfetta L. Femoral neck preservation in total hip replacement. Ital J Orthop Traumatol 1993; 19(1): 5-12.

16. Albanese C V, Santori F S, Pavan L, Learmonth I D, Passariello R. Periprosthetic DXA after total hip arthroplasty with short vs. ultrashort custom-made femoral stems. Acta Orthop 2009; 80(3): 291-7. doi: $10.3109 / 17453670903074467$.

17. Salemyr M, Muren O, Ahl T, Bodén H, Eisler T, Stark A, et al. Lower periprosthetic bone loss and good fixation of an ultra-short stem compared to a conventional stem in uncemented total hip arthroplasty. Acta Orthop 2015; 86(6): 659-66. doi: 10.3109/17453674.2015.1067087.

18. Christiansen J D, Ejaz A, Nielsen P T, Laursen M. An ultra-short femoral neck-preserving hip prosthesis: a 2-year follow-up study with radiostereometric analysis and dual $\mathrm{x}$-ray absorptiometry in a stepwise introduction. J Bone Joint Surg Am 2020; 102(2): 128-36. doi: 10.2106/ JBJS.19.00104.

19. Gillies R M, Kohan L, Cordingley R. Periprosthetic bone remodelling of a collum femoris preserving cementless titanium femoral hip replacement. Comput Methods Biomech Biomed Engin 2007; 10(2): 97-102. doi: 10.1080/10255840601003577.

20. Kress A M, Schmidt R, Nowak T E, Nowak M, Haeberle L, Forst $\mathbf{R}$, et al. Stress-related femoral cortical and cancellous bone density loss after collum femoris preserving uncemented total hip arthroplasty: a prospective 7-year follow-up with quantitative computed tomography. Arch Orthop Trauma Surg 2012; 132(8): 1111-19. doi: 10.1007/s00402-0121537-0.

21. Kim Y-H, Park J-W. Long-term outcomes of ultra-short metaphysealfitting anatomic cementless femoral stem in total hip arthroplasty with ceramic-on-ceramic articulation for young patients. J Arthroplasty 2019; 34(10): 2427-33. doi: 10.1016/j.arth.2019.04.036.

22. Hutt J, Harb Z, Gill I, Kashif F, Miller J, Dodd M. Ten year results of the collum femoris preserving total hip replacement: a prospective cohort study of seventy five patients. Int Orthop 2014; 38(5): 917-22. doi: 10.1007/s00264-013-2212-y.

23. Formica M, Cavagnaro L, Basso M, Zanirato A, Palermo A, Felli L. What is the fate of the neck after a collum femoris preserving prosthesis? A nineteen years single center experience. Int Orthop 2017; 41(7): 132935. doi: 10.1007/s00264-016-3350-9.

24. Klein L J, Puretic G, Mohaddes M, Kärrholm J. Similar clinical results and early subsidence between the Collum Femoris Preserving and the Corail stem: a randomized radiostereometric study of 77 hips with 2 years' follow-up. Acta Orthop 2019; 90(3): 202-8. doi: 10.1080/17453674.2019.1577344

25. Kärrholm J. Radiostereometric analysis of early implant migration: a valuable tool to ensure proper introduction of new implants. Acta Orthop 2012; 83(6): 551-2. doi: 10.3109/17453674.2012.745352. 
26. Babu S, Singh P, Wiik A, Shastri O, Malik K, Bailey J, et al. A comparison of patient-reported outcome measures (PROMs) between short and conventional stem hip replacements: a systematic review and meta-analysis. HIP Int 2019; 112070001988821. doi: 10.1177/1120700019888210.

27. Graves S. Australian Orthopaedic Association National Joint Replacement Registry (AOANJRR). Hip, knee \& shoulder arthroplasty: 2019 annual report. Adelaide: AOA; 2019.

28. Lidder S, Epstein D J, Scott G. A systematic review of short metaphyseal loading cementless stems in hip arthroplasty. Bone Joint J 2019; 101B(5): 502-11. doi: 10.1302/0301-620X.101B5.BJJ-2018-1199.R1.

29. Kendoff D O, Citak M, Egidy C C, O'Loughlin P F, Gehrke T. Eleven-year results of the anatomic coated CFP stem in primary total hip arthroplasty. J Arthroplasty 2013; 28(6): 1047-51. doi: 10.1016/j. arth.2012.10.013.

30. Wacha H, Domsel G, Herrmann E. Long-term follow-up of 1217 con- secutive short-stem total hip arthroplasty (THA): a retrospective singlecenter experience. Eur J Trauma Emerg Surg 2018; 44(3): 457-469. doi: 10.1007/s00068-017-0895-2.

31. Kärrholm J, Rogmark C, Nauclér E, Vinblad J, Mohaddes M, Rolfson O. The Swedish Hip Arthroplasty Register Annual Report 2018. The Swedish Hip Arthroplasty Register; 2019 p. 9557296 byte. doi: 10.18158/ HKQHN6SL8.

32. Valstar E R, Gill R, Ryd L, Flivik G, Börlin N, Kärrholm J. Guidelines for standardization of radiostereometry (RSA) of implants. Acta Orthop 2005; 76(4): 563-72. doi: 10.1080/17453670510041574.

33. Yan S G, Li D, Yin S, Hua X, Tang J, Schmidutz F. Periprosthetic bone remodeling of short cementless femoral stems in primary total hip arthroplasty: a systematic review and meta-analysis of randomized-controlled trials. Medicine 2017; 96(47): e8806. doi: 10.1097/ MD.0000000000008806. 